\title{
Laparoendoscopic Single-Site Surgery na realização de criptorquidectomia laparoscópica em um gato
}

\author{
Laparoendoscopic Single-Site Surgery in performing laparoscopic cryptorchidectomy in a cat
}

\author{
João Pedro Scussel Feranti ${ }^{\mathrm{I}}$ Marília Teresa de Oliveira ${ }^{\mathrm{I}}$ Fernando Wiecheteck de Souza \\ Luís Felipe Dutra Corrêa ${ }^{I}$ Saulo Tadeu Lemos Pinto Filho ${ }^{I}$ Fabíola Dalmolin ${ }^{I}$ \\ Hellen Fialho Hartmann ${ }^{\mathrm{I}}$ Maurício Veloso Brun ${ }^{\mathrm{I}^{*}}$
}

\section{- NOTA -}

RESUMO

A afecção congênita mais comum nos animais domésticos é denominada de criptorquidismo, sendo caracterizada pela não migração de um ou ambos os testículos até o escroto. $O$ objetivo deste trabalho é relatar a técnica de criptorquidectomia videoassistida, através de um único acesso (LESS) sem dispositivos especificos, em um gato siamês com dois anos de idade e $3,9 \mathrm{~kg}$ de massa corporal. Utilizou-se um único portal de $5 \mathrm{~mm}$, inserido na linha média ventral na região pré-púbica. Por esse portal, a cavidade foi insuflada com $\mathrm{CO}_{2}$ medicinal, permitindo a obtenção do pneumoperitônio $(8 \mathrm{mmHg})$ a uma velocidade de $1 \mathrm{l} / \mathrm{min}$. Após o inventário da cavidade abdominal, com o auxilio de um endoscópio de $5 \mathrm{~mm}$ e $25^{\circ}$, identificou-se o testículo ectópico. Com a utilização de uma pinça de apreensão para mini laparoscopia (3mm), introduzida ao lado do trocarte (pelo mesmo acesso), o testículo foi fixado e tracionado para o exterior da cavidade abdominal para realização de hemostasia, não sendo necessária a ampliação da ferida cirúrgica. Após a secção dos vasos testiculares, o testículo foi totalmente liberado e o coto vascular devolvido à cavidade abdominal. O presente relato demonstra que a cirurgia laparoscópica por um único acesso (LESS) pode ser utilizada com sucesso no diagnóstico e tratamento de casos de criptorquidismo em gatos, com as possíveis vantagens que o acesso apresenta em relação à cirurgia convencional.

Palavras-chave: orquiectomia, videocirurgia, felino, LESS.

\section{ABSTRACT}

The most common congenital disease in domestic animals is cryptorchidism, which is characterized by the failure of one or both testicles to descend into the scrotum. The objective of this paper is to describe a video-assisted cryptorchidectomy through a single access (LESS), without specific portal multifunctional, in a two-year-old siamese cat weighing $3.9 \mathrm{~kg}$. To perform the cryptorchidectomy, it was inserted a single portal $(5 \mathrm{~mm})$ in the ventral midline in the pre-pubic region. The cavity was insufflated with medicinal $\mathrm{CO}_{2}$ at a rate of $11 /$ minute in order to obtain a pneumoperitoneum (8mmHg). After the inspection of the abdominal cavity with an endoscope $\left(5 \mathrm{~mm} / 25^{\circ}\right)$ the ectopic testicle was identified. With a minilaparoscopy, forceps (3mm) was introduced in the side of the first portal (same access), the testicle was grasped and pulled outside to realize hemostasis, without need to extend surgical wound. After sectioning the testicular vessels, the testicle was fully released and the vascular stump was returned to the abdominal cavity. The present report demonstrates that laparoscopic surgery by a single access (LESS) may be successful in the diagnosis and treatment of cryptorchidism in cats, considering the potential benefits that this procedure brings in relation to conventional surgery.

Key words: orchiectomy, videosurgery, feline, LESS.

A afecção congênita mais comum nos animais domésticos é denominada de criptorquidismo, sendo caracterizada pela não migração de um ou ambos os testículos até o escroto. $O$ órgão retido pode ser encontrado na cavidade abdominal, no canal inguinal ou no subcutâneo, perto do escroto, sendo sua ocorrência uni ou bilateral (AMANN \& VEERAMACHANENI, 2007). O criptorquidismo unilateral é mais frequente quando comparado ao bilateral, e o testículo direito é o mais acometido. Animais criptorquídicos unilaterais podem ter a fertilidade reduzida, já os bilaterais são funcionalmente estéreis (MANSFIELD \& LAND, 2002).

Ao realizar cirurgias do trato reprodutivo, a laparoscopia é uma alternativa à laparotomia, pois oferece uma adequada visualização das

'Departamento de Clínica de Pequenos Animais (DCPA), Faculdade de Medicina Veterinária, Centro de Ciências Rurais (CCR), Universidade Federal de Santa Maria (UFSM), 97105-900, Av. Roraima, 1000, Cidade Universitária, Camobi, Santa Maria, RS, Brasil. E-mail: mauriciovelosobrun@hotmail.com. *Autor para correspondência. 
estruturas anatômicas por uma diminuto acesso. A criptorquidectomia laparoscópica é considerada uma técnica segura, de fácil e rápida execução (VANNOZZI, 2002; PROOT, 2007).

A cirurgia laparoscópica através de um único acesso (LESS) consiste em uma evolução natural da aplicação da cirurgia minimamente invasiva. Modificações e refinamentos dos instrumentais laparoscópicos têm promovido crescimento substancial, principalmente na área da urologia, nos últimos anos (FARACO, 2012). Como a NOTES (Natural Orifice Transluminal Endoscopic Surgery), a LESS (Laparoendoscopic Single-site Surgery) representa o último avanço da cirurgia minimamente invasiva, devido à diminuição das complicações associadas à utilização de um único portal, diminuindo o desconforto e promovendo melhor aspecto estético em relação à laparoscopia convencional (SYMES \& RAINE, 2011).

O objetivo deste trabalho é relatar a técnica de criptorquidectomia videoassistida através de um único acesso (LESS), em um gato criptorquida. Segundo o conhecimento dos autores, tal condição ainda não havia sido descrita na medicina de pequenos animais.

Um gato siamês com dois anos de idade e $3,9 \mathrm{~kg}$ de massa corporal foi encaminhado ao Hospital Veterinário Universitário da Universidade Federal de Santa Maria (HVU-UFSM), tendo como relato do proprietário a castração há aproximadamente um ano. Contudo, o proprietário referia comportamento compatível como de macho adulto inteiro, com demarcação de território, micção frequente e fugas constantes. Foram palpados o escroto, a região dos canais inguinais, o tecido subcutâneo e a região pré-escrotal, não sendo evidenciados testículos. Presumiu-se, então, a possibilidade de localização intra-abdominal de um dos testículos, o qual não havia sido extirpado durante a primeira operação.

Ao exame clínico, constataram-se mucosas rosadas, temperatura de $38,6^{\circ} \mathrm{C}$, frequência cardíaca de 140 batimentos $/$ minuto $^{-1}$, frequência respiratória de 24 movimentos $/$ minuto $^{-1}$ e tempo de perfusão capilar menor que dois segundos. O animal apresentavase em bom estado nutricional. Foram efetuados hemograma completo e provas de bioquímica sérica, compreendendo mensurações de creatinina, ureia, ALT e albumina, estando todos os valores dentro dos referenciais para a espécie. Realizou-se ecografia abdominal, evidenciando-se imagem compatível com testículo intracavitário.

Após o diagnóstico de criptorquidia, optouse pela realização da criptorquidectomia videocirúrgica por um único acesso. Como medicação pré-anestésica, foi administrada a associação de cetamina (10 $\mathrm{mg} \mathrm{kg}^{-1}$, I.M.), midazolam (0,4mg kg-1 , I.M.) e morfina $(0,3$ $\mathrm{mg} \mathrm{kg}{ }^{-1}$, I.M.). A indução anestésica foi obtida com a aplicação de propofol ( $3 \mathrm{mg} \mathrm{kg}^{-1}$, I.V.) e a manutenção com isoflurano vaporizado em $\mathrm{O}_{2}$ a $100 \%$, em circuito semiaberto. Após a indução anestésica, administrouse ampicilina sódica (20mg $\mathrm{kg}^{-1}$, I.V.) como quimioprofilaxia antimicrobiana.

O animal foi colocado em decúbito dorsal e, após a antissepsia do campo operatório, realizouse a introdução do trocarte $(5 \mathrm{~mm})$ por meio de uma incisão na pele e linha média ventral, na região prépúbica (técnica aberta). Por esse portal, a cavidade foi insuflada com $\mathrm{CO}_{2}$ medicinal, permitindo a obtenção do pneumoperitônio ( $8 \mathrm{mmHg}$ ) a uma velocidade de 11/ min. Após o inventário da cavidade abdominal, com o auxílio de um endoscópio de $5 \mathrm{~mm}$ e $25^{\circ}$, identificou-se o testículo ectópico (Figura 1A). Com a utilização de uma pinça de apreensão para mini laparoscopia (3mm), introduzida ao lado do trocarte (pelo mesmo acesso), o testículo foi apreendido (Figura 1B) e tracionado para o exterior da cavidade abdominal, não sendo necessária a ampliação da ferida cirúrgica (Figura 1C).

Por meio de cirurgia convencional, empregando-se a técnica das três pinças, foi promovida hemostasia e secção dos vasos testiculares e do ducto deferente (Figura 1D), utilizandose uma ligadura circular e outra transfixante, com poliglactina 910 3-0. Após a secção dessas estruturas, o testículo foi totalmente liberado e o coto vascular devolvido à cavidade abdominal. Ao fim do procedimento, foi realizada a inspeção do abdome, confirmando-se ausência de hemorragia. $\mathrm{O}$ trocarte foi retirado, seguindo-se com a desinsuflação da cavidade abdominal e sutura da lesão de acesso do portal. Os tecidos muscular e subcutâneo foram ocluídos em padrão Sultan com poliglactina 910 3-0, e a pele, em padrão interrompido simples com náilon monofilamentar 4-0.

No pós-operatório, foi realizada limpeza da ferida (t.i.d.) com solução de $\mathrm{NaCl}$ a $0,9 \%$. Administrou-se meloxicam (0,1 $\mathrm{mg} \mathrm{kg}^{-1}$, V.O., s.i.d.) e dipirona ( $25 \mathrm{mg} \mathrm{kg}^{-1}$, V.O., b.i.d.) durante três dias.

$\mathrm{O}$ procedimento cirúrgico durou 30 minutos e a única intercorrência observada foi uma lesão cutânea adicional ao lado do acesso, promovida durante a execução da técnica aberta de introdução do primeiro portal. Tal lesão foi suturada e cicatrizou por primeira intenção.

Devido à escassez de estudos referentes à comparação das técnicas (LESS x laparoscopia convencional), ainda não se pode quantificar a superioridade da LESS. Porém, aventa-se que tal procedimento poderia apresentar algumas potenciais 


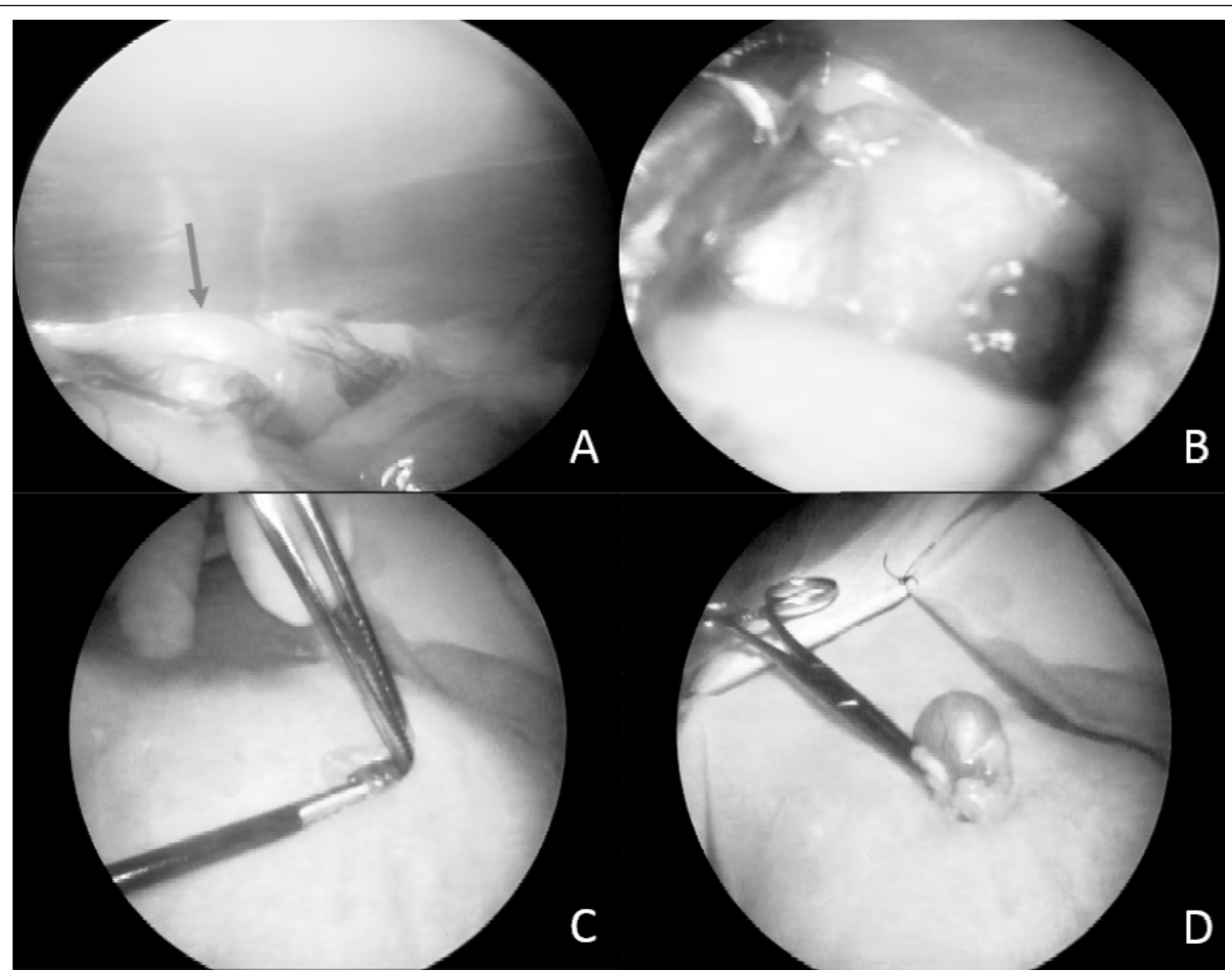

Figura 1 - Principais etapas do procedimento cirúrgico de criptorquidectomia laparoscópica em um gato siamês, com dois anos de idade e $3,9 \mathrm{~kg}$ de massa corpórea. (A) Identificação do testículo ectópico (seta), após o inventário da cavidade abdominal com o auxílio de um endoscópio de $5 \mathrm{~mm}$ e $25^{\circ}$. (B) Fixação do testículo com auxílio de pinça de apreensão pediátrica para mini laparoscopia $(3 \mathrm{~mm})$. (C) Exteriorização do testículo com auxílio de pinça de apreensão pediátrica de $3 \mathrm{~mm}$, sem necessidade de ampliação da ferida de acesso. (D) Testículo exteriorizado para posterior hemostasia e secção dos vasos testiculares e do ducto deferente.

vantagens, tais como: menor incidência de infecções, perda sanguínea, formação de enfisema subcutâneo e morbidade, acarretando em recuperação mais rápida do paciente, além de melhor aspecto cosmético (KYONG-SHIL, 2011; OLWENY, 2012). Todavia, dependendo da condição do paciente, o uso da LESS pode não ser recomendado, como em animais obesos, em condições clínicas inadequadas e na vigência de aderências intra-abdominais ou hérnia diafragmática (MATYJASIK, 2011).

Alguns autores recomendam o estabelecimento do pneumoperitônio através da agulha de Veress (PENA et al., 1998; PROOT, 2007), porém, neste caso, optou-se pela técnica aberta com o intuito de minimizar a possibilidade de lesões iatrogênicas relacionadas ao uso da agulha.

A execução da LESS, comparativamente a outras técnicas videocirúrgicas onde se utilizam outros portais para instrumentação e não um único portal para a óptica e os instrumentais, resulta em dificuldades, principalmente em relação à manipulação dos instrumentos, por se trabalhar com estes em paralelismo com o endoscópio, prejudicando o campo visual e ocasionando contato entre estes. Outras dificuldades da técnica foram semelhantes às verificadas em demais procedimentos laparoscópicos com mais portais, como a ausência de sensação tátil, imagem bidimensional e a limitação de movimentos, as quais tendem a ser superadas com o treinamento da equipe e a ascendência na curva de aprendizado.

Como a pinça para mini laparoscopia foi introduzida na cavidade abdominal pela mesma incisão de acesso na qual foi colocado o trocarte (ao lado deste), a manobra de apreensão do testículo e posterior exteriorização teve que ser realizada rapidamente, devido ao escape de gás pela ferida parietal. Ainda assim, 
tal manobra não provocou enfisema subcutâneo. Após a exteriorização do testículo e dos vasos testiculares, optouse pela hemostasia deles pela técnica aberta, por ser considerada rápida e de fácil execução, não dependendo de outro método mais oneroso ou dependente de equipamentos específicos como Ligasure $^{\mathrm{TM}}$, bisturi ultrassônico, clipes e/ou eletrocoagulação.

Além de seu ineditismo, o presente relato permite supor que a LESS é uma alternativa menos invasiva que a cirurgia convencional, com potencial lesivo inferior ao da cirurgia videoassistida com dois portais (OLIVEIRA et al., 2011). Possibilita, ainda, demonstrar que a cirurgia videoassistida por um único acesso (LESS) pode ser utilizada com sucesso no diagnóstico e tratamento de casos de criptorquidismo em gatos, com as possíveis vantagens que o acesso demonstra em relação à cirurgia convencional.

\section{COMITÊ DE ÉTICA E BIOSSEGURANÇA}

O relato foi executado com animal atendido na rotina do HVU-UFSM e não foi parte de qualquer projeto, sendo apenas um relato de caso clínico. Os autores ficam à disposição para quaisquer esclarecimentos futuros sobre o trabalho.

\section{REFERÊNCIAS}

AMANN, R.P.; VEERAMACHANENI, D.N. Cryptorquidismin common eutherian mammals. Reproduction, Cambridge, v.133, n.3, p.141-161, 2007. Disponível em: <http://www.reproductiononline.org/content/133/3/541.long >. Acesso em: 21 abr. 2015. doi: 10.1530/rep-06-0272.

FARACO, C.S. Avaliação do Centry Port como acesso único (Less Laparoscopic Single Sight Surgery) na realização de criptorquidectomia laparoscópica: estudo experimental em coelhos: (Oryctolagus cuniculus). 2012. 89f. Dissertação (Mestrado em Ciências Veterinárias na área de morfologia, cirurgia e patologia) - Faculdade de Veterinária, UFRGS, RS.

KYONG-SHIL, I.M. et al. Laparoscopic Single-site surgery for adnexal tumors: a comparasion of surgical outcomes and postoperative pain outcomes. Kaohsiung Journal of Medical Sciences, Taiwan, v.27, n.3, p.91-95, 2011. Disponível em: <http://
www.sciencedirect.com/science/article/pii/S1607551X10000331>. Acesso em: 20 abr. 2015. doi: 10.1016/j.kjms.2010.10.001.

MANSFIELD, K.G.; LAND, E.D. Cryptorchidism in florida panthers: prevalence, features, and influence of genetic restoration. Journal Wild Disease, Kansas, v.38, p.693-698, 2002. Disponível em: <http://www.ncbi.nlm.nih.gov/pubmed/12528434>. Acesso em: 21 abr. 2015. doi: 10.7589/0090-3558-38.4.693.

MATYJASIK, H. et al. Laparoscopic procedures in dogs and cats. Publish Journal of Veterinary Sciences, Olsztyn, v.14, n.2, p.305-316, 2011. Disponível em: <http:/www.ncbi.nlm.nih.gov/ pubmed/21721421>. Acesso em: 20 abr. 2015.

OLWENY, E.O. et al. Importance of cosmesis to patients undergoing renal surgery: a comparision of Laparoendoscopic Single-Site (LESS), laparoscopic and open surgery. BJU International, Oxford, v.110, n.2, p.268-272, 2012. Disponível em: <http://onlinelibrary. wiley.com/doi/10.1111/j.1464-410X.2011.10784.x/epdf>. Acesso em: 20 abr. 2015. doi: 10.1111/j.1464-410X.2011.10784.x.

OLIVEIRA, R.P. et al. Criptorquidectomia bilateral vídeo-assistida em um gato - relato de caso. Revista Científica de Medicina Veterinária. Pequenos Animais e Animais de Estimação, Curitiba, v.29, p.220-224, 2011.

PENA, F.J. et al. Laparoscopic surgery in a clinical case of seminoma in a cryptorchid dog. Veterinary Record, London, v.142, p.671-672, 1998. Disponível em: <http://veterinaryrecord. bmj.com/content/142/24/671.full.pdf + html $>$. Acesso em: 21 abr. 2015. doi: 10.1136/vr.142.24.671.

PROOT, J. Laparoscopy-assisted cryptorchidectomy in the dog and cat. Companion Animal, UK, v.12, p.1-4, 2007. Disponível em: <http://www.readcube.com/articles/10.1111\% 2Fj.2044-3862.2007.tb00212>. Acesso em: 20 abr. 2015. doi: 10.1111/j.2044-3862.2007.tb00212.x.

SYMES, A.; RANE, A. Urological applications of Single-site laparoscopic surgery. Journal of Minimal Access Surgery, Mumbai, v.7, n.1, p.90-95, 2011. Disponível em: <http://www. ncbi.nlm.nih.gov/pmc/articles/PMC3002016>. Acesso em: 21 abr. 2015. doi: 10.4103/0972-9941.72394.

VANNOZZI, I. et al. Laparoscopic cryptorchidectomy in a cat. Journal of Feline Medicine and Surgery, UK, v.4, p.201-203, 2002. Disponível em: <http://jfm.sagepub.com/content/4/4/201. full.pdf + html $>$. Acesso em: 20 abr. 2015. doi: 10.1053/ jfms.2002.0168 